TIPA. Travaux interdisciplinaires sur la parole et le langage

28 | 2012

Linguistique expérimentale

\title{
Mesure du potentiel des tâches d'interaction. Expérimentation et perspectives méthodologiques
}

\section{Elena Cosereanu-Declerck}

\section{(2) OpenEdition}

Journals

Édition électronique

URL : http://journals.openedition.org/tipa/203

DOI : 10.4000/tipa.203

ISSN : 2264-7082

Éditeur

Laboratoire Parole et Langage

Référence électronique

Elena Cosereanu-Declerck, « Mesure du potentiel des tâches d'interaction. Expérimentation et perspectives méthodologiques », TIPA. Travaux interdisciplinaires sur la parole et le langage [En ligne], 28 | 2012, mis en ligne le 13 septembre 2012, consulté le 01 mai 2019. URL : http:// journals.openedition.org/tipa/203 ; DOI : 10.4000/tipa.203

Ce document a été généré automatiquement le 1 mai 2019.

La revue TIPA. Travaux interdisciplinaires sur la parole et le langage est mise à disposition selon les termes de la licence Creative Commons Attribution - Pas d'Utilisation Commerciale - Pas de Modification 4.0 International. 


\title{
Mesure du potentiel des tâches d'interaction. Expérimentation et perspectives méthodologiques
}

\author{
Elena Cosereanu-Declerck
}

\section{Introduction}

1 Si la plupart des chercheurs travaillant sur l'acquisition des langues secondes (L2) ${ }^{1}$ s'accordent aujourd'hui à penser que la pratique de la communication reste une condition sine qua non pour que l'apprenant puisse développer et parfaire ses compétences, la manière dont cette activité contribue à ce développement reste massivement hypothétique et fait l'objet de nombreuses controverses. Parmi la multitude complexe de structures d'interaction susceptibles de jalonner les communications en L2 où s'implique l'apprenant (que son interlocuteur soit natif ou non-natif), les épisodes de négociation (du sens et de la forme) et les épisodes de correction ont reçu une attention toute particulière dans la recherche en acquisition des langues (RAL). En effet, plusieurs chercheurs, tant du côté francophone que du côté anglo-saxon, voient dans ces structures un levier déterminant pour l'acquisition. La valeur des tâches d'interaction communicationnelle pour le développement des compétences de l'apprenant en L2 (et ainsi leur valeur pédagogique), pourrait en bonne partie s'expliquer par leur capacité à susciter l'apparition de tels épisodes de négociation ou de correction..

2 Sur la base de cette hypothèse, nous avons conduit une série d'études expérimentales destinées à évaluer le potentiel acquisitionnel de différentes tâches d'interaction, réalisées avec des apprenants de français langue étrangère (FLE) en binômes, sous différentes conditions de communication synchrone (clavardage - chat - et face-à-face). Nous avons pour cela mesuré leur capacité à susciter des épisodes de négociation et de rétroaction corrective dans des séquences potentiellement acquisitionnelles. 
3 Après avoir rappelé les principes du paradigme interactionniste en RAL, et sa conception du rôle de la correction et des tâches (section 1), nous présentons les principes de notre étude expérimentale (section 2), ainsi qu'une synthèse (section 3) et une discussion des résultats (section 4). Nous proposons finalement une discussion générale sur le rôle que l'expérimentation et les analyses quantitatives sont susceptibles de jouer dans la RAL et la didactique des L2 (section 5). Nous défendons à ce titre l'idée que les analyses des productions langagières que propose la RAL ne doivent pas se restreindre à des approches qualitatives et descriptives, mais qu'elles doivent également être de nature quantitative.

\section{Paradigme interactionniste, épisodes de correction et tâches d'interaction}

4 L'approche interactionniste de l'acquisition consiste, dans son principe, à tenir la position que l'interaction - et la dimension sociale qui lui est inhérente - est constitutive des processus cognitifs, savoirs et savoir-faire sur lesquels repose la compétence linguistique de l'apprenant (Arditty \& Vasseur, 1999 : 12 ; Pekarek, 2000). L'interaction n'est donc pas réductible à un simple cadre qui fournirait des données langagières et permettrait de déclencher ou d'accélérer certains processus développementaux, mais elle constitue un facteur structurant le processus même de ce développement (Pekarek, 2000). Pekarek (1999) a ainsi proposé d'identifier trois postulats qui forment le cœur de l'approche interactionniste : 1) le rôle constitutif de l'interaction pour le développement langagier; 2) la sensibilité contextuelle des compétences langagières; 3) la nature située et réciproque de l'activité discursive (et cognitive).

5 Une idée généralement défendue dans ce cadre, en particulier depuis l'hypothèse de l'interaction de Long (1983), est qu'une situation susceptible de donner lieu à l'apprentissage sera une situation permettant à l'apprenant de prendre conscience d'un écart ou d'une inadéquation entre son interlangue / répertoire linguistique (la représentation, la connaissance ou l'élaboration partielle, provisoire et en évolution que l'apprenant a de la L2) et la L2 telle qu'elle lui est présentée par un référent externe. En général, il s'agit d'un locuteur expert en qui l'apprenant a confiance. Les corrections auxquelles est alors exposé l'apprenant quand il produit des énoncés en L2 favoriseraient ainsi le processus d'acquisition dans l'exacte mesure où elles lui permettraient de prendre conscience de ces écarts et de les résorber (soit en modifiant des hypothèses nonconformes, soit en en acquérant de nouvelles) ${ }^{2}$. Une fonction déterminante de la correction serait par conséquent d'expliciter à l'apprenant des règles et connaissances sur lesquelles sa pratique de la langue s'appuie, mais dont il ne lui est pas forcément aisé, voire dont il ne lui est pas possible, de prendre conscience.

6 Une manière de formaliser la fonction de la correction dans l'acquisition est de recourir aux notions de témoignage positif et négatif ${ }^{3}$. Le témoignage positif (positive evidence) se définit comme une production qui fournit à l'apprenant un modèle des énoncés possibles et grammaticalement corrects dans la langue cible. Le témoignage négatif (negative evidence) indique, quant à lui, à l'apprenant les énoncés grammaticalement incorrects ou inacceptables dans la L2 (Schachter, 1984 ; White, 1989, 1996 ; Long, 1996 ; Braidi, 2002). L'hypothèse selon laquelle la rétroaction corrective est susceptible d'amener l'apprenant à prendre conscience des points d'inadéquation/écart entre son interlangue et la L2, met au premier plan la fonction de témoignage négatif que peut endosser la rétroaction. On 
peut en effet défendre l'idée d'une insuffisance de la seule exposition de l'apprenant au témoignage positif pour le développement de sa L2 (voir par exemple Long \& Robinson, 1998). Comme l'expliquent Lyster \& Mori (2006), il est important que les apprenants bénéficient d'une information sur le succès communicationnel de leur utilisation de la langue cible. Ces auteurs l'expriment en ces termes:

«[les apprenants] pourraient avoir besoin d'une rétroaction sur leurs erreurs quand ils ne sont pas capables de découvrir, par la seule exposition à l'évidence positive, les points où leur interlangue diffère de la langue-cible » (270).

7 Si l'exposition de l'apprenant à la correction est tenue pour favoriser l'acquisition ${ }^{4}$, la question se pose toutefois de savoir quels sont les facteurs qui rendent une correction efficace pour une restructuration utile du système interlinguistique de l'apprenant. Il semble en effet évident que toute correction n'est pas génératrice d'acquisition. Et des facteurs comme l'attention jouent probablement une fonction centrale dans le processus qui va rendre la correction utilisable pour une restructuration de l'interlangue (Schmidt \& Frota, 1986 ; Schmidt, 1990, 1995 ; Hulstijn, 1989a, 1989b ; R. Ellis, 1994 ; Long \& Robinson, 1998 ; Narcy-Combes, 2005).

8 Une hypothèse, discutée mais prometteuse, défendue par certains tenants de la théorie interactionniste, consiste à poser qu'une correction ne va en général être efficace - c'està-dire pouvoir donner lieu à une modification du système de l'interlangue de l'apprenant - que si elle est intégrée dans des épisodes d'interaction communicationnelle particuliers qu'on appelle "interactions négociées ", ou "épisodes de négociation», ou encore " épisodes relatifs au langage » (langage related episodes). Ces épisodes communicationnels sont pour cette raison qualifiés de « séquences potentiellement acquisitionnelles » (SPA) (Py et al., 1988 ; De Pietro et al., 1989; Py, 1990). L'interaction négociée se définit comme une interaction où un ou plusieurs signaux d'incompréhension donnent lieu, par l'intermédiaire d'une rétroaction corrective, à une résolution des énoncés problématiques. On catégorise parfois ces épisodes communicationnels selon qu'ils sont focalisés sur la forme ou sur le sens. Pica (1994: 56) a ainsi décrit l'interaction négociée, quand elle porte sur le sens (ce qu'elle appelle la négociation du sens), comme une activité dans laquelle les apprenants cherchent la clarification, la confirmation, et la répétition d'énoncés en L2 qu'ils ne comprennent pas 5 .

Les interactions négociées constitueraient notamment des conditions propices pour l'acquisition d'une L2, parce qu'elles poussent les apprenants à opérer des changements dans leur output langagier. L'apprenant exposé à la rétroaction corrective pourrait non seulement prendre conscience des inadéquations/écarts entre son interlangue et la L2, mais encore restructurer ou compléter celle-ci afin de pallier ces écarts (Gass, 1997 ; Long, 1996 ; Pica, 1994 ; R. Ellis, 2003) ${ }^{6}$. Il faut également mentionner à ce titre l'importance de la réaction de l'apprenant aux rétroactions correctives, en particulier de l'incorporation, soit l'utilisation par l'apprenant exposé à une rétroaction corrective de la forme corrigée par son interlocuteur pour former un nouvel énoncé. L'incorporation pourrait en effet témoigner : d'une part que l'apprenant exposé à la rétroaction corrective était bien animé d'un niveau d'attention suffisant pour prendre conscience de la correction qui lui était adressée - fonction décisive du repérage (noticing) dans le traitement de l'input - ; d'autre part, qu'il s'est bel et bien approprié la forme corrigée, qu'il l'a bien intégrée à son répertoire linguistique propre, puisqu'il en fait désormais usage.

La valeur des tâches d'interaction communicationnelle, notamment des tâches d'écart d'informations ${ }^{7}$, pour le développement des compétences de l'apprenant en L2 (et ainsi 
leur valeur pédagogique), pourrait en bonne partie s'expliquer par leur capacité à susciter l'apparition de telles structures d'interaction. Les tâches d'interaction sont des tâches dont la réalisation implique par définition une interaction communicationnelle, et elles engagent donc a minima deux individus. Ce type de tâche se réalise à travers la mise en place d'une communication orale ou écrite, et demande par exemple que les locuteurs collaborent afin d'atteindre un objectif fixé au préalable (décrire des images, raconter une histoire, utiliser une carte, etc.). L'usage que les apprenants font du langage a, dans ce cas, pour finalité première de communiquer: s'exprimer et se faire comprendre par l'interlocuteur. Or, ce type d'activité communicationnelle pourrait sous certaines conditions favoriser la mise en place d'épisodes de négociation et de correction (R. Ellis, 2003 ; Pellettieri, 1999). Plus généralement, en installant l'apprenant dans une situation d'interaction communicationnelle fonctionnellement analogue à une situation d'interaction naturelle, la tâche d'interaction pourrait contribuer à un développement efficace de ses compétences en L2, car le conduisant à mobiliser ses savoirs et savoir-faire afin de mettre en place une forme de communication authentique. Une caractéristique remarquable des tâches d'interaction est en effet que les structures de la L2 ne sont pas rencontrées de façon décontextualisée (c'est-à-dire de manière abstraite vis-à-vis de leur pouvoir communicationnel : ce qu'elles permettent de «dire» ou de «faire»), mais au contraire saisies dans leur portée sémantique concrète et communicationnelle ellemême : c'est depuis ce qu'elles permettent de signifier qu'elles sont envisagées, dans le cadre d'une tentative d'intercompréhension authentique. Elles pourraient ainsi constituer un outil pédagogique de première importance dans l'enseignement/ apprentissage des L2.

11 Le chapitre $7 \mathrm{du} \mathrm{CECR}^{8}$ (« Les tâches et leur rôle dans l'enseignement des langues »), est consacré à la notion de tâche et aux caractéristiques et usages des tâches. Dans la même perspective, Springer (2009) rappelle ce qui suit :

«les tâches pédagogiques communicatives (contrairement aux exercices formels hors contexte) visent à impliquer l'apprenant dans une communication réelle, ont un sens (pour l'apprenant), sont pertinentes (ici et maintenant dans la situation formelle d'apprentissage), exigeantes mais faisables (avec un réajustement de l'activité si nécessaire) et ont un résultat identifiable » (29).

Différentes études empiriques ont ainsi montré que les tâches d'interaction (en particulier de tâches d'écarts d'information) permettaient d'exposer l'apprenant à des situations d'usage de la L2 dont la valeur acquisitionnelle est largement reconnue, en particulier: exposition à un input riche, situation d'usage de la L2 authentique, c'est-àdire polarisée par la finalité de communiquer et de résoudre un problème concret, nécessité de produire un output compréhensible, exposition à des séquences de communication impliquant de la négociation du sens, de la forme/des formes, des épisodes de correction, etc. La question de savoir quels types de tâches maximisent ce développement, et quelles dimensions de ces tâches, et plus encore du contexte où elles se réalisent, décident de leur potentiel acquisitionnel, est pourtant aujourd'hui encore une question largement ouverte.

13 C'est pour y apporter des éléments de réponse que nous avons conduit, dans la continuité de différents travaux (Prabhu, 1987 ; Pica, 1994 ; Smith, 2003 ; R. Ellis, 2003 ; Gass et al., 2005), plusieurs études expérimentales. L'ambition première de ces études est d'identifier des tâches et des situations et contextes de réalisation de ces tâches, susceptibles de maximiser le développement des connaissances et compétences de l'apprenant en L2. Il s'agit, qui plus est, de tenter d'apercevoir la complexité des phénomènes auxquels nous 
sommes ici confrontés, et en particulier de mettre au jour les facteurs susceptibles de décider de la valeur de l'activité par tâche pour l'acquisition.

Compte tenu du format réduit du présent article, et parce que nous avons déjà présenté et discuté ailleurs ces études ${ }^{9}$, nous n'en présentons ici qu'un échantillon. Nous nous focalisons sur une étude qui visait à comparer le potentiel acquisitionnel de plusieurs tâches d'écarts d'informations en mesurant leur capacité à susciter des épisodes de rétroaction corrective et de négociation.

\section{Protocole expérimental et hypothèses de travail}

15 L'étude expérimentale ici présentée consistait à comparer différents types de tâches d'écart d'information réalisées en binôme, par clavardage ${ }^{10} /$ chat ou à l'oral, afin de déterminer : 1) si celles-ci présentent une potentialité différente à générer des types d'interaction langagière susceptibles de favoriser le processus d'acquisition (en particulier: routines de négociation et rétroactions correctives), et 2) le cas échéant, quels caractères de ces tâches pourraient expliquer ces différences.

étude comprenait deux expériences organisées suivant des principes analogues : des binômes d'apprenants de FLE avaient pour consigne de réaliser trois types de tâches dans deux modalités communicationnelles : clavardage/chat et oral en face-à-face, soit un total de six tâches par binôme. Afin d'éviter que la même tâche ne soit répétée dans les deux modalités communicationnelles, chaque type de tâche comportait un contenu différent pour le clavardage et l'oral: le principe de la tâche restait exactement le même, le contenu seul variait.

L'expérience A comportait 30 apprenants volontaires de FLE (organisés en 15 binômes), d'âge moyen 22,9 ans et de 15 nationalités différentes. L'expérience B comportait 12 apprenants volontaires de FLE (organisés en 6 binômes), d'âge moyen 21,1 ans et de 7 nationalités différentes. Les participants étaient des étudiants ingénieurs de l'Université de Technologie de Compiègne inscrits dans des UV de FLE. Les deux expériences furent réalisées au Centre de Langues de l'Université de Technologie de Compiègne : l'expérience A pendant le semestre de printemps 2007 (au mois d'avril), l'expérience B pendant le mois d'août 2007, à l'occasion d'un stage intensif de FLE. Elles eurent lieu en dehors des cours proprement dits. Tous les apprenants étaient volontaires.

18 Avant l'expérience, chaque apprenant compléta la grille d'auto-évaluation du C.E.C.R. de Strasbourg (publié en 2001) ${ }^{11}$, ce qui nous permit d'attribuer à chacun un indice de niveau (indice s'échelonnant comme suit, par niveau croissant de maitrise de la langue : A1, A2, B1, B2, C1, C2). Les questionnaires firent apparaitre que les participants avaient un niveau en FLE variable : le niveau des apprenants de l'expérience $\mathrm{A}$ allait de $\mathrm{A} 2$ à $\mathrm{C} 1$; celui des apprenants de l'expérience $\mathrm{B}$ allait de $\mathrm{A} 1$ à $\mathrm{B} 2$.

Les groupes d'apprenants de l'expérience $\mathrm{A}$ et de l'expérience $\mathrm{B}$ n'étaient donc pas totalement homogènes. Outre des différences de nationalité, et une légère différence d'âge, les apprenants des deux groupes n'étaient dans l'ensemble pas de même niveau, et - point sur lequel nous reviendrons plus loin - leur démarche d'apprentissage n'était sans doute pas non plus soutenue par le même degré de motivation.

Il importe de le préciser car la finalité de cette étude étant de mesurer et comparer le potentiel acquisitionnel de tâches d'interactions, à partir de leur capacité à générer des épisodes de négociation et de correction, il est nécessaire, dans la mesure du possible, de 
stabiliser les variables susceptibles d'influer sur ces indicateurs. En vérité, c'est principalement pour des raisons d'ordre pratique que nous n'avons pas pu homogénéiser totalement les deux groupes: il s'agissait de groupes d'apprenants volontaires dans le cadre d'un stage intensif ou de cours de FLE à l'Université. Nous n'étions pas en mesure de construire ces groupes nous-même.

Que les groupes de l'expérience $\mathrm{A}$ et de l'expérience B ne soient pas homogènes n'est cependant pas un problème, la comparaison du potentiel acquisitionnel des tâches menée ici ayant d'abord lieu à l'intérieur d'un même groupe. Ce sont les mêmes binômes d'apprenants qui réalisent les trois types de tâches, ce qui autorise à attribuer les différences communicationnelles observées à la structure et aux conditions de réalisation des tâches, et non à une différence dans le profil des apprenants. La comparaison entre les deux expériences permet de son côté d'évaluer dans quelle mesure d'autres facteurs que la nature et les conditions de réalisation des tâches, entrent en ligne de compte dans la détermination de leur potentiel acquisitionnel.

Pour l'expérience $\mathrm{B}$, nous avons également demandé aux participants de remplir un questionnaire destiné à évaluer leur attitude personnelle à l'égard des tâches réalisées, et visant notamment à évaluer leur perception de la facilité ou de la difficulté des tâches, dans les modalités comparées. Nous n'en rapportons toutefois pas ici les résultats, lesquels ont déjà été décrits et discutés dans un autre travail ${ }^{12}$.

Les tâches d'interaction utilisées étaient les suivantes :

- Raconter une scène d'un film (notée tâche "film" dans la suite) : chaque membre du binôme se voit présenter une séquence d'un film français de comédie, l'un des deux apprenants visionnant la scène avec le son (en utilisant des écouteurs), alors que l'autre ne bénéficie que de l'image (pas d'écouteurs). Après avoir visionné la séquence, les apprenants doivent communiquer de manière à parvenir à un accord sur les événements du film (tâche d'écart d'information pouvant en ce sens également être considérée comme une tâche d'écart d'opinion).

- Trouver les différences entre deux images apparemment identiques (notée tâche "image" dans la suite): chaque participant se voit attribuer une image d'un couple d'images similaires mais présentant quelques différences, et il a pour consigne de dialoguer avec son partenaire de manière à identifier les détails qui différencient leurs deux images. Aucune indication n'est donnée aux participants sur le nombre de différences entre les deux images (au total 10 pour la série d'images utilisées par clavardage, et 8 pour la série d'images utilisées à l'oral).

- Résoudre une énigme à partir d'une série d'énoncés textuels (notée tâche "énigme" dans la suite) : chaque étudiant se voit attribuer trois des six indices qui composent une énigme, et a pour consigne de dialoguer avec son partenaire de manière à la résoudre (tâche d'écart d'information qu'on peut en ce sens également considérer comme une tâche d'écart de raisonnement) $)^{13}$.

24 Si l'on reprend la typologie de Prabhu $(1987)^{14}$, on peut considérer que ces trois tâches sont des tâches d'écart d'information: les apprenants ne disposent pas des mêmes informations, et doivent mettre en commun leurs informations pour résoudre la tâche. Toutefois, la tâche "film" peut également être considérée comme une tâche d'écart d'opinion, dans la mesure où les deux apprenants sont amenés à exprimer leur opinion sur le passage du film visionné. Et la tâche "énigme" peut également être considérée comme une tâche d'écart de raisonnement, au sens où elle possède une forte composante en termes d'activités de raisonnement logique. Elle pourrait également être considérée 
comme une tâche d'écart d'opinion, car les apprenants sont amenés à donner leur opinion sur la solution logique de l'énigme, même si celle-ci ne comporte en définitive qu'une seule solution.

Pour les sessions de communication par clavardage, nous avons utilisé le logiciel Net Meeting. Les participants ont réalisé des sessions d'entraînement de quelques minutes pour se familiariser avec le logiciel, avant de passer à la résolution des tâches proprement dites. Dans la condition clavardage, les participants avaient pour consigne exprès de ne pas dialoguer à l'oral et de ne pas avoir recours aux signes iconiques (du type émoticônes) mis à disposition par le logiciel. Pour les sessions de communication à l'oral en face-àface, chaque participant était installé d'un côté d'une table, à approximativement 1 mètre 50 de son interlocuteur. Pour la tâche de comparaison des images, chaque participant tenait ses images dans ses mains de manière que son interlocuteur - de toute façon $a$ priori trop éloigné pour en distinguer les détails - ne puisse en percevoir le contenu. Les communications par clavardage ont été enregistrées sous forme numérique (via des fichiers au format html) et les communications orales ont été enregistrées, au format mp3 , à l'aide d'un dictaphone numérique. Les communications orales enregistrées ont ensuite été transcrites et vérifiées.

L'hypothèse théorique générale, de facture interactionniste, qui sous-tend cette étude est que l'exposition de l'apprenant à la rétroaction corrective, ou son exposition à des épisodes d'interaction négociée, peuvent favoriser l'amélioration de ses connaissances, et le cas échéant de ses compétences communicationnelles en L2 (Gass, 1997 ; Long, 1996 ; Pica, 1994 ; R. Ellis, 2003). C'est sur la base de cette hypothèse que nous avons défini les indicateurs utilisés pour évaluer le potentiel acquisitionnel des tâches d'interaction comparées. Selon ce principe d'évaluation, une tâche qui suscite la génération d'un nombre d'interactions négociées et de rétroactions correctives plus important qu'une autre pourra être considérée comme possédant un potentiel acquisitionnel plus important: sa réalisation par l'apprenant sera plus susceptible de développer ses connaissances/compétences en L2. Ce principe d'évaluation peut être considéré comme une application de l'hypothèse de l'interaction de Long (1983). Cette hypothèse affirme, dans son principe, que plus il y a d'opportunités de négociation, plus l'acquisition est probable (voir par exemple Benoît, 2004).

\section{Résultats et discussion}

Afin de déterminer si les trois tâches décrites, ainsi que les deux modalités de communication étudiées, étaient plus ou moins adaptées pour la génération de séquences d'interaction susceptibles de favoriser l'acquisition, nous avons analysé les communications à l'aide d'une grille des types d'interactions communicationnelles, et avons comptabilisé leur nombre par tâche et par modalité pour chaque communication. Nous ne présentons ici qu'une partie de ces résultats : à savoir le nombre de rétroactions correctives et d'épisodes de négociation. Nous avons ainsi mesuré de nombreux autres indicateurs, comme le phénomène d'évitement de l'erreur, l'appropriation des corrections (incorporation) ou les transferts interlinguistiques, pour n'en citer que quelques-uns. Cette restriction se justifie par l'idée que la rétroaction corrective et la négociation sont deux indicateurs privilégiés du processus d'acquisition (voir section 1). Ils peuvent en ce sens suffire pour quantifier le potentiel acquisitionnel des tâches étudiées. Nous examinons uniquement ici les effets du type de tâche (et, dans une certaine 
mesure, de la composition des binômes d'apprenants, notamment leur degré de symétrie en termes de niveaux de compétence dans la L2), non les effets de la dimension communicationnelle ou d'autres facteurs, sur les interactions ${ }^{15}$.

\section{1 Épisodes de négociation} tâche "énigme" entre deux étudiants vietnamiens, M.P. et S.T. (de niveaux respectifs B1 et B1/B2), contient une routine de négociation simple, de nature lexicale, centrée sur l'explication du sens du mot «bilingue », que M.P. déclare ignorer (tour T3) :

T1 M.P. : et 6) : leroux est bilingue ! [Déclencheur lexical / Début de la routine de négociation]

T2 S.T. : ok!

T3 $\underline{\text { M.P. }}$ : mais je ne sais pas le mot : bilingue [Indicateur lexical]

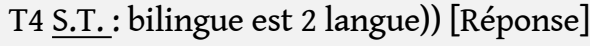

S.T. : par exemple

S.T. : anglais et français

S.T. : alors, M.leroux enseigne Anglais et français

S.T. : et c'est tout avec lui

L'épisode de communication suivant, tiré d'une communication par clavardage entre un apprenant chinois (C.Z.) et un apprenant vietnamien (H.N.), de niveaux respectifs A1 et A2, comprend deux routines de négociations simples qui se suivent, et chacune est résolue par un recours à une marque transcodique, soit un emprunt de matériel linguistique à une autre langue que le français pour communiquer : ici, l'anglais.

Tab. 1. Exemples de routines de négociation tirées du corpus de l'expérience

T1 C.Z. : et je vois un homme était content, pourquoi ? [Déclencheur lexical]

T2 $\underline{\text { H.N. }}$ : content, Qu'est-ce que c'est ? [Indicateur lexical]

T3 C.Z. : contant

T4 $\underline{\text { H.N. }}$ : Je ne comprends pas

T5 C.Z. : happy [Réponse / Marque transcodique de L3]

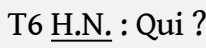

H.N. : Je ne vois personne

TIPA. Travaux interdisciplinaires sur la parole et le langage, 28 | 2012 
T7 C.Z. : le personne qui était le plus chic [Déclencheur lexical]

T8 $\underline{\text { H.N. }}$ : Chic, Qu'est-ce que c'est ? [Indicateur lexical]

T9 C.Z. : handsome [Réponse / Marque transcodique de L3]

T10 H.N. : Non

T11 C.Z. : beau [Réponse 2]

C.Z. : oui [Réaction à la réponse minimale]

L'analyse statistique des données (analyse de la variance, notée ANOVA dans la suite) ne permet pas de déterminer s'il y a un effet de la tâche sur le nombre de routines de négociation générées dans les communications (ANOVA pour l'expérience $A: p=0,44$; pour l'expérience $B: p=0,41$ ). Nous n'avons pas non plus observé d'effet statistiquement significatif de la tâche sur le nombre de tours de négociations. Précisons toutefois que la fréquence de tours de négociation générés dans les communications de l'expérience $B$ était plus élevée que dans celles de l'expérience A (Fig. 1). En effet, si l'on calcule le pourcentage de tours de négociation dans les deux expériences (en rapportant le nombre de tours de négociation au nombre total de tours de parole), on voit qu'il est largement inférieur dans l'expérience A (voir Figure 2 ci-dessous).

Fig. 1. Pourcentage moyen de tours de négociation par modalité et par expérience

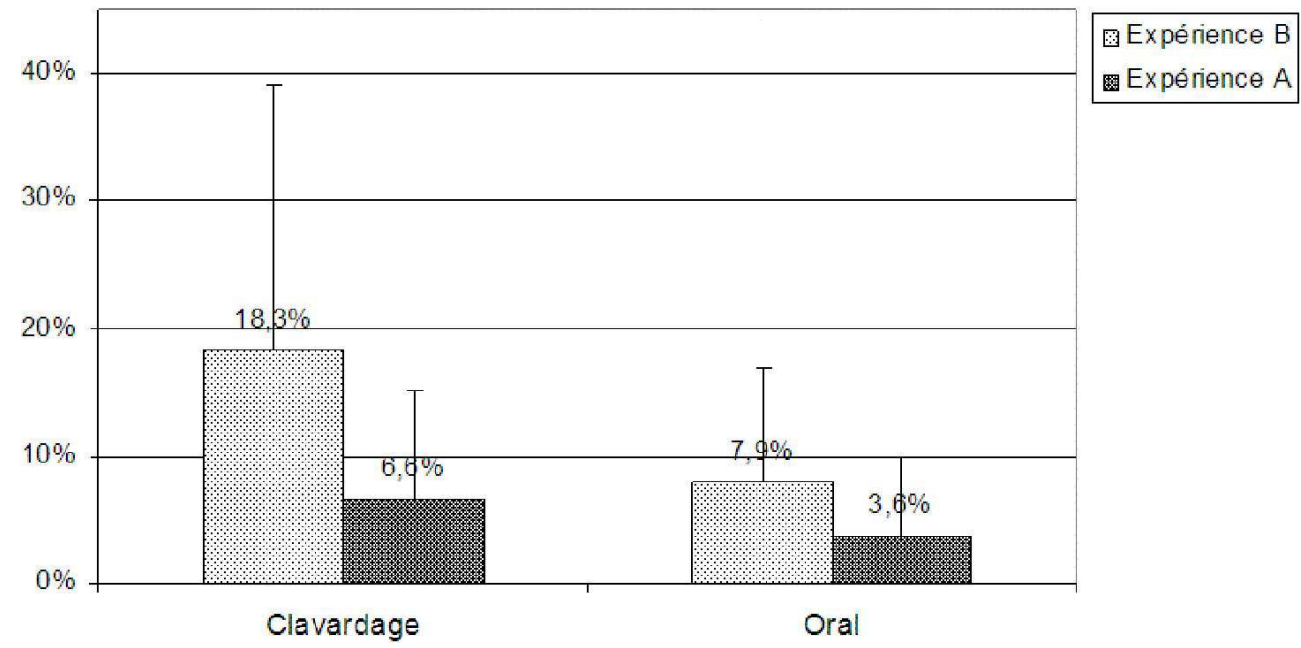

Cette différence importante dans le nombre d'interactions négociées entre les apprenants des deux expériences pourrait en partie s'expliquer par des facteurs motivationnels : alors que les apprenants de l'expérience B étaient en stage intensif de FLE, et se trouvaient depuis très peu de temps en France (pour certains depuis seulement quelques semaines), et ont par ailleurs manifesté des indices comportementaux certains de motivation, les apprenants de l'expérience $\mathrm{A}$ étaient depuis plusieurs mois au moins déjà en France, en fin de semestre universitaire, et en période d'examen.

Elle pourrait également s'expliquer - ce n'est toutefois pas une possibilité alternative au point précédent, mais plutôt complémentaire - par une différence d'attitude communicationnelle des apprenants qui sont depuis plus longtemps immergés en milieu linguistique français. Le besoin de corriger l'interlocuteur se ferait moins sentir au fur et à mesure que l'apprenant se familiariserait avec le milieu linguistique et la culture de la langue cible. 

apprenants peut également nous apporter certaines pistes de réponse pour expliquer cette différence. Nous l'avons expliqué plus haut, nous avons mesuré le niveau des apprenants en utilisant la grille d'auto-évaluation du C.E.C.R. ${ }^{16}$. Nous avons associé à chaque niveau un chiffre ( $\mathrm{A} 1=1 ; \mathrm{A} 2=2 ; \mathrm{B} 1=3 ; \mathrm{B} 2=4 ; \mathrm{C} 1=5 ; \mathrm{C} 2=6)$ et avons calculé sur cette base le niveau moyen des apprenants des deux expériences. Ces calculs ont fait apparaître que les apprenants de l'expérience $B$ avaient en moyenne un niveau intermédiaire entre A2 et B1 (indice calculé $=2,6$ ), et que ceux de l'expérience A avaient en moyenne un niveau légèrement supérieur à $\mathrm{B} 1$ (indice calculé = 3,15).

Nous avons également cherché à quantifier la différence de niveau entre les étudiants composant les différents binômes, lesquels étaient plus ou moins symétriques. En effet, nous n'avons pas imposé aux apprenants la composition des binômes, nous les avons laissé décider eux-mêmes de leur partenaire. Pour ce faire, nous avons repris les indices numériques précédents, et nous avons calculé la différence d'indice entre chacun des deux apprenants composant les binômes. Nos calculs ont fait apparaitre que les binômes de l'expérience B étaient en moyenne composés d'apprenants ayant une différence de niveau s'approchant de deux échelons (indice calculé $=0,8$ ). Alors que les binômes de l'expérience A étaient en moyenne composés d'apprenants ayant une différence de niveau légèrement supérieure à un échelon (indice calculé $=0,6$ ).

Cette différence dans les profils des apprenants des deux expériences, et dans le degré de symétrie/dissymétrie des binômes, pourrait en partie expliquer les différences observées dans la production d'interactions négociées. On sait en effet que les communications exolingues constituent généralement un terrain plus propice à la production d'interactions négociées que les communications endolingues (Bange, 1987; De Pietro, 1988; Py et al., 1988 ; Véronique 1992, 2000 ; Porquier \& Py, 2004 ; Behrent, 2007 ; Véronique et al., 2009). En particulier, il s'agit de situations asymétriques où apparait fréquemment ce que Py et al. (1988) appellent un contrat didactique (voir également Porquier \& Py, 2004 ; Véronique, 1992, 2000), soit une forme d'entente implicite ou explicite se mettant en place entre le locuteur natif (LN) et le locuteur non natif (LNN), prenant la forme d'un étayage au sens de Bruner (1983), et où le LN va endosser la fonction de tuteur vis-à-vis du LNN. Ce type de situation d'étayage semble également pouvoir s'installer entre deux LNN, s'il s'agit d'un locuteur novice et d'un locuteur expert, le second endossant le rôle de tuteur pour le premier.

38 Sur cette base, il nous semble raisonnable de faire l'hypothèse que plus un binôme d'apprenants présentera une dissymétrie importante, plus une telle situation d'étayage par contrat didactique sera susceptible de se mettre en place - et plus les interactions réalisées auront de chance de comporter des épisodes de négociation. On peut en effet penser que l'apparition d'un contrat didactique va faciliter la réalisation de routines de négociation, en particulier au sens où les locuteurs produiront plus facilement des demandes de clarification ou des signaux de non-compréhension s'ils perçoivent qu'ils évoluent dans ce qu'on pourrait appeler un « climat de confiance ». différences observées dans le nombre de tours de négociation en moyenne générés dans les communications respectives des deux expériences. Les binômes de l'expérience $B$ étant globalement plus dissymétriques que ceux de l'expérience $A$, des situations d'étayage se seraient plus facilement réalisées, d'où une fréquence d'apparition de tours de négociation accrue.

TIPA. Travaux interdisciplinaires sur la parole et le langage, 28 | 2012 

ici qu'à titre d'hypothèses explicatives. D'autres études empiriques, ciblées sur ces points spécifiques (et investiguant notamment l'influence de la dissymétrie des binômes sur les fréquences de production d'interactions négociées), seraient nécessaires pour apporter des éléments de réponse plus systématiques sur ces questions.

\subsection{Rétroactions correctives}

41 lors des communications, et les avons distinguées selon leur caractère explicite ou implicite, conformes aux normes de la L2 ou non, et selon leur nature autocorrective. Nous laissons toutefois ici de côté les autocorrections, qui sont fonctionnellement différentes des autres formes de rétroactions correctives, puisqu'elles ne sont pas dirigées vers les énoncés de l'interlocuteur, mais vers les énoncés personnels de l'apprenant. Les rétroactions correctives ont également été catégorisées selon leur nature lexicale, grammaticale ou phonétique, les rétroactions phonétiques ne concernant bien entendu que les communications orales.

Dans l'épisode de communication suivant, tiré d'une communication par clavardage sur la tâche "film" entre deux apprenants de nationalité canadienne, A.C. (de niveau B2) et B.P. (de niveau C1), B.P. adresse deux rétroactions correctives (RC) explicites à A.C., en lui spécifiant clairement que ses productions sont non conformes à la L2, et en lui énonçant explicitement la règle qui s'applique en français $(\mathrm{T} 2)^{17}$ :

T1 A.C. : Après la repas mauvais, l'enquêteur a changé tes vêtements dans un taxi et il se plain avec le conducteur [Production non-conforme grammaticale] T2 B.P. : Il faut mettre l'adjectif mauvais devant le mot repas. [RC explicite grammaticale 1]

[...]

T3 B.P. : C'est plutôt 'ses vêtements' et non 'tes vêtements'. [RC explicite grammaticale 2]

Dans cet exemple, tiré d'une communication orale sur la tâche "image", l'apprenant italien A.C. (de niveau A2) ne connaît pas le mot « Noël» en français et utilise la forme telle qu'elle existe dans sa langue maternelle, l'italien : «natale ». La correction de la part de son interlocuteur, S.V. (de nationalité belge et de niveau B2), est produite immédiatement et de manière implicite.

T1 A.C. : J'ai compris ! Ah, j'ai compris ! And eh... Et eh...Sur eh...il a le eh...bret...pour natale ! [Production non-conforme de nature lexicale / Emprunt à la L1] T2 S.V. : Noël ? Non, pas chez moi ! [Réparation lexicale]

Lors des communications orales en face-à-face, les étudiants peuvent également produire des énoncés phonologiquement non-conformes à la L2. Ces productions non-conformes sont le plus souvent corrigées de manière implicite (c'est-à-dire via une réparation).

Dans l'épisode de communication suivant, tiré d'une communication orale sur la tâche "film", les deux apprenants chinois Z.L. et R.D. (respectivement de niveaux A2 et B1) communiquent pour décrire la scène du film visionnée. L'apprenant Z.L. ne prononce pas

TIPA. Travaux interdisciplinaires sur la parole et le langage, 28 | 2012 
correctement le mot «vin » et R.D. le corrige de manière implicite, en lui fournissant une réparation phonétique dans le tour suivant.

Tab. 2. Exemples de rétroactions correctives tirées du corpus de l'expérience

T1 Z.L. : le vine et le eh... [Production non-conforme de nature phonétique]

T2 $\underline{\text { R.D. }}$ : oui, oui, le vin ! le vin et le [incompréhensible] ? Je pense eh...c'est tout?

Est-ce qu'il y a sans part...qu'on a pas? [Réparation phonétique]

Si l'on additionne les rétroactions correctives implicites et explicites, conformes aux normes de la L2 ou non, on observe un effet significatif de la tâche sur le nombre moyen de rétroactions correctives générées lors des communications. On le voit sur la Figure 4 ci-dessous, les résultats indiquent que la tâche "image" est plus efficace que les deux autres pour générer des rétroactions correctives. Elle est suivie par la tâche "film", puis par la tâche "énigme".

Fig. 2. Nombre moyen de rétroactions correctives
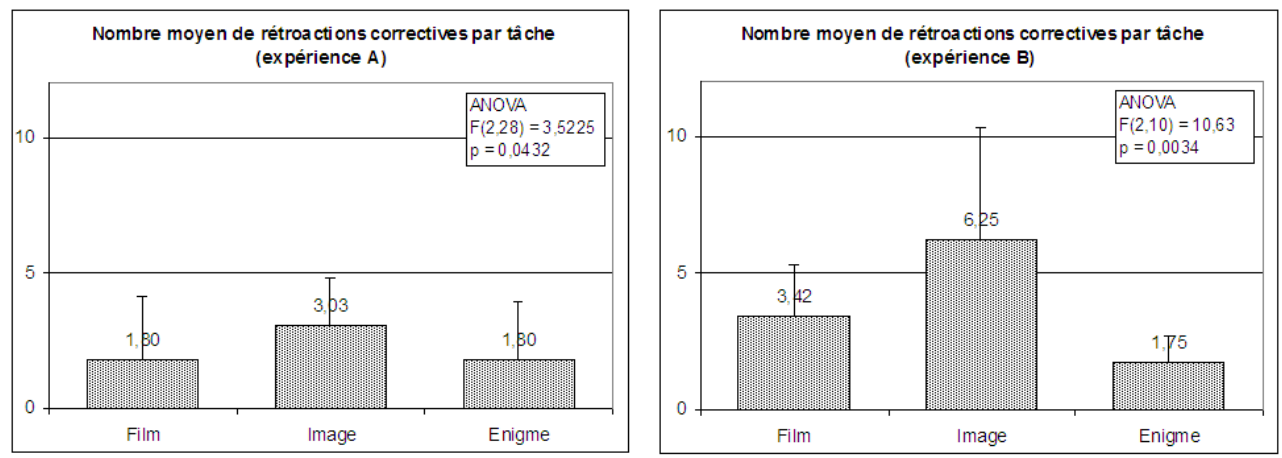

Remarquons également que, comme on peut le voir en comparant les deux graphiques de la Figure 4, beaucoup moins de rétroactions correctives ont été générées lors des communications de l'expérience A que lors de celles de l'expérience B (en moyenne deux fois moins de rétroactions dans l'expérience $A$ ). Cette fois encore, cette différence nous semble pouvoir en partie s'expliquer par des différences dans les structures des binômes des deux expériences. Les binômes de l'expérience $B$ étant globalement plus dissymétriques que ceux de l'expérience $\mathrm{A}$, des situations d'étayage se seraient plus facilement mises en place, d'où une fréquence d'apparition de rétroactions correctives accrue.

Nous avons ainsi observé que dans des communications entre des apprenants dont l'écart de niveau était important (par exemple des apprenants de niveau B2 et de niveau C1), l'apprenant expert endossait souvent la fonction de tuteur à travers un processus d'étayage déployé de manière systématique à chaque production non-conforme de son interlocuteur (les rétroactions correctives sont ainsi pratiquement toutes explicites) ${ }^{18}$. A l'inverse, chez des apprenants de même niveau, il était fréquent que les productions non conformes d'un apprenant ne donnent lieu à aucune rétroaction corrective alors même que l'interlocuteur avait connaissance de la forme correcte (comme en témoignait la suite de la communication).

Ainsi, dans l'épisode de communication par clavardage suivant, sur la tâche "énigme", les apprenants X.T. et T.S.P., qui sont de même niveau (B1) et tous deux de nationalité 
vietnamienne, la production non-conforme de X.T. sur le mot matieres (omission de l'accent grave) ne donne pas lieu à une rétroaction corrective explicite de la part de T.S.P., alors même que celui-ci connaît la forme correcte du mot, comme en témoigne l'usage qu'il en fait dans la seconde réplique du tour de parole T4. Bien que sur un plan purement fonctionnel, il s'agisse bien d'une rétroaction corrective implicite, cette réplique ne semble pas avoir pour finalité de corriger X.T., mais uniquement de poursuivre le fil de la communication. Du fait de cette absence de démarche de correction explicite et d'étayage (aucun contrat didactique ne se met en place), X.T. persiste dans son usage incorrect de la forme linguistique, comme on peut le voir dans la seconde réplique du tour de parole T5.

T1 $\underline{\text { X.T. }}$ : et a la fin chacun enseigne trois matieres [Production non-conforme lexicale]

T2 T.S.P. : oui [RC non saisie]

T3 $\underline{\text { X.T. }}$ : vu et vous

T4 T.S.P. : d'accord

T.S.P. : aucune matière n'est enseigneé par 3 personnes [Réparation lexicale]

T5 X.T. : vu oui

$\underline{X . T .}$ : vu est ce que chaque matiere est enseigné par deux persons [Répétition de la production non-conforme]

On retrouve le même phénomène dans l'épisode de communication par clavardage qui suit, autour de la tâche "image", entre les apprenants W.X. et Z.L., de même niveau (B1) et tous deux de nationalité et de langue maternelle chinoise, à propos du mot gauche: la production non-conforme de W.X. qui écrit gouche au tour de parole T1 ne donne pas lieu à une correction explicite de la part de Z.L., qui utilise pourtant la forme correcte du mot dans la deuxième réplique du tour de parole 4. W.X. semble toutefois avoir aperçu la correction dans la mesure où il fait cette fois usage de la forme correcte du mot gauche au tour de parole T9.

T1 W.X. : sur première étagère il y a deux tasses à gouche [Production nonconforme lexicale]

T2 Z.L. : pour du vin?

Z.L. : ou pour du café?

T3 W.X. : et il y a deux "'"'"'"'" pour café à droite

T4 Z.L. : oui

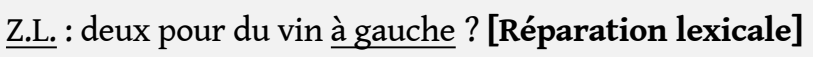

T5 W.X. : Oui

T6 Z.L. : Continue

T7 W.X. : d' accord

T8 Z.L. : donc

Z.L. : le rideau..

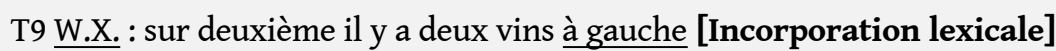

51 Notons toutefois que la différence de niveau entre apprenants, prise isolément, n'est pas suffisante pour expliquer la mise en place du processus d'étayage et son importance. Il semble également nécessaire de tenir compte du profil des apprenants, notamment de 
leur " personnalité » (cf. Véronique, 1997 ; Dewaele \& Furnham, 2004), mais également de leur culture éducative (cf. M-F. Narcy-Combes, 2010).

\section{Discussion générale sur le potentiel acquisitionnel des tâches}

Tout d'abord, concernant ce que nous proposons ici d'appeler la dimension collaborative : la tâche "image" semble être celle des trois tâches qui exige le degré de collaboration le plus important pour être résolue: dans la mesure où chacun des deux partenaires a besoin que l'autre lui décrive précisément le contenu de son image, il ne peut opérer seul. La tâche "film" apparaît à ce titre comme présentant une dimension collaborative moindre: ce point a notamment trait au fait que son enjeu est beaucoup moins clairement défini que dans la tâche "image", puisque la consigne exhorte simplement les participants à raconter à leur interlocuteur ce qu'ils ont compris du passage du film visionné : ainsi, il n'y a pas de problème précis à résoudre, pas d'objectif pragmatique clairement défini, même s'il est minimalement requis des participants de parvenir à un terrain d'entente sur le sens de la séquence visionnée. Ainsi, alors que la tâche "image" possède un caractère fermé: elle n'a qu'une seule solution, la tâche "film" est intrinsèquement ouverte: elle possède de multiples solutions. Enfin, la tâche "énigme" semble être celle dont le degré de collaboration exigé est le plus faible, puisque rien n'interdit aux participants de livrer d'emblée leurs indices à leur partenaire. Il était ainsi fréquent de voir les participants livrer en bloc dès le début de l'interaction leurs énoncés. Au fond, la tâche "énigme" n'est donc une tâche d'écart d'information qu'aussi longtemps que les apprenants n'ont pas accès aux indices de leur interlocuteur : elle devient une tâche d'écart d'opinions dès lors que les deux apprenants du binôme se sont transmis leurs indices, et ont ainsi sous les yeux le même contenu textuel.

Ces réflexions rejoignent la distinction proposée par Gass et al. (2005: 582) entre les tâches d'échange optionnel d'information (la tâche peut, en principe, être résolue sans échange d'information) et les tâches d'échange d'information requis (la tâche ne peut être résolue sans échange). Dans leur étude comparative sur trois tâches, une tâche d'échange optionnel d'information et deux tâches d'échange d'information requis, Gass et al. (2005)

TIPA. Travaux interdisciplinaires sur la parole et le langage, 28 | 2012 
ont ainsi observé que la réalisation de la tâche d'échange optionnel d'information donnait lieu à la production d'une quantité d'interactions négociées largement inférieure aux deux tâches d'échange d'information requis (cf. Gass et al., 2005 : 590).

Concernant les différences de contenu entre les différentes tâches, on peut remarquer que la tâche "image" contient des éléments iconographiques et non pas du texte, alors que la tâche "énigme" possède a contrario un contenu uniquement textuel. La tâche "film" est de ce point de vue à mi-chemin entre les deux, bien qu'il ne s'agisse pas de texte mais de matériel linguistique oral. Il faut également remarquer à ce propos que la tâche "image" exige la traduction de l'information iconographique en une information linguistique, qu'elle soit textuelle ou orale, pour sa transmissibilité. De même, la tâche "film" requiert la traduction d'une information visuelle, à savoir le passage du film visionné, en matériel linguistique, et il y intervient notamment une composante narrative.

On peut également remarquer que la tâche "image" est celle des trois tâches étudiées qui exige la plus grande précision descriptive. L'identification des différences entre les deux images oblige en effet à une précision lexicale importante, et pour le moins suffisante (elle peut très bien reposer sur l'usage de paraphrases si l'apprenant ignore le terme qu'il veut signifier à son interlocuteur) pour que l'apprenant puisse comprendre à quel élément iconographique de son image son interlocuteur fait référence. Ce caractère est en revanche beaucoup moins prégnant dans les deux autres tâches.

Ces différences de contenu et de modalités de réalisation des différentes tâches pourraient en partie expliquer les différences observées dans le nombre et la fréquence des types d'interaction langagière que nous avons étudiés précédemment. En particulier, le fait que la tâche "film" et la tâche "image" présentent une fréquence de génération de rétroactions correctives plus importante par rapport à la tâche "énigme" nous semble clairement pouvoir s'expliquer par ces caractéristiques. Le fait que la tâche "énigme" n'exige pas la traduction d'une information non linguistique, par exemple iconographique, dans un format linguistique ${ }^{20}$ et qu'elle peut être résolue par chacun des participants de manière isolée, une fois les indices de l'autre obtenus, pourrait expliquer que la communication s'accompagne de moins de demandes de clarification et de rétroactions visant à préciser le contenu sémantique du message de l'interlocuteur.

On doit toutefois noter, en ce qui concerne le critère de collaboration, que d'autres études empiriques ne semblent à première vue pas appuyer cette hypothèse. Ainsi, Smith (2003) a mené une étude comparative sur deux types de tâches: une tâche de type puzzle, consistant à remettre en ordre des images désordonnées, et une tâche de type prise de décision, où des objets doivent être appariés à des personnes. Et il a observé que la tâche de "prise de décision" générait plus d'interactions négociées ( $44 \%$ du nombre de tours de parole total) que la tâche "puzzle" ( $23 \%$ du nombre de tours de parole total). Or, comme il le fait remarquer, dans la continuité des principes de description des tâches proposés par Pica et al. (1993), la tâche de "prise de décision" diffère de la tâche "puzzle", en ce que sa résolution n'exige pas nécessairement de collaboration avec l'interlocuteur ${ }^{21}$. On est dans une situation proche de la tâche "film" et de la tâche "énigme" de nos études. L'objectif de la tâche "film" est en effet suffisamment peu contraignant pour qu'il y ait une pression collaborative pour sa résolution : ainsi, rien n'empêche que l'un des deux apprenants du binôme prenne en charge la quasi totalité de la description du passage du film visionné, l'autre apprenant pouvant se contenter de lui manifester qu'il est d'accord avec sa version. Et la tâche "énigme" a la spécificité de pouvoir être résolue de manière individuelle, dès lors qu'un des apprenants a accès aux informations de son 
interlocuteur : les informations ayant déjà une forme textuelle, l'apprenant peut très bien les transmettre directement à son interlocuteur.

Ce résultat de Smith semble donc aller à l'encontre de nos propres observations, ainsi qu'à l'encontre des prédictions de Pica et al. (1993) qui postulaient, comme le note Smith (2003 : 45) lui-même, que les tâches de type "puzzle" devraient faciliter la négociation plus que les tâches d'écart d'information, de résolution de problème, de prise de décision ou d'échange d'opinion.

61 Cette différence pourrait toutefois venir, comme l'a suggéré Smith (2003), des items lexicaux que l'auteur avait insérés dans les tâches, procédé auquel nous n'avons pour notre part pas eu recours. Smith a en effet inséré dans ses tâches des items lexicaux cibles, que les apprenants ne maîtrisaient pas, partant de l'hypothèse que ces items faciliteraient le déclenchement d'épisodes de négociations. Or, il a observé que si, en globalité, la tâche de "prise de décision" générait plus de négociation que la tâche "puzzle", en revanche, cette dernière générait plus de négociations que la tâche de "prise de décision" si l'on ne prenait en considération que les items déclencheurs ne correspondant pas aux items-cibles (cf. Smith, 2003 : 46). La tâche "puzzle" aurait donc la spécificité de générer plus de négociations incidentes, alors que la tâche de "prise de décision" aurait un plus fort potentiel à générer des interactions négociées sur les itemscibles spécialement insérés dans la tâche (items a priori inconnus ou faiblement maîtrisés par les apprenants). Une hypothèse explicative avancée par Smith est que les items lexicaux cibles insérés dans les tâches pourraient avoir été perçus comme secondaires dans la tâche "puzzle", mais pas dans la tâche de "prise de décision".

62 A l'issue de ces différentes réflexions, on peut faire les propositions suivantes sur les caractères de la tâche qui sont susceptibles d'augmenter son potentiel acquisitionnel ${ }^{22}$ :

- sa résolution doit exiger une réelle collaboration de la part des membres du binôme: la tâche ne doit pas pouvoir être résolue seul ;

- les informations à partager pour la résolution de la tâche ne doivent pas être immédiatement transmissibles, mais un jeu de va-et-vient doit au contraire s'installer. Ce caractère rejoint en un sens le premier, puisque si l'information est immédiatement transmissible, la tâche pourra être résolue de manière isolée une fois les informations de l'interlocuteur obtenues;

- la résolution de la tâche doit exiger une grande précision descriptive. Ce facteur peut en effet favoriser la production de rétroactions correctives visant le contenu sémantique, et plus généralement la génération de demandes de clarification.

63 Notons que le second point évoqué dans cette liste rejoint l'insistance mise par R. Ellis sur la nécessité que les tâches comportent chaque fois une forme d'écart (gap). L'écart force en effet les apprenants à interagir, tout en maintenant la polarisation de la communication sur l'objectif pragmatique de la tâche, et maximise les probabilités que des épisodes d'interaction comme les routines de négociation se mettent en place ${ }^{23}$.

Ces différentes hypothèses sur les critères susceptibles de favoriser le potentiel acquisitionnel des tâches permettent de faire des prédictions expérimentales qui pourraient être mises à l'épreuve à travers d'autres études empiriques. Nous n'avons pour notre part pas eu l'occasion de le faire, mais nous pouvons énoncer ici une série de principes généraux qui pourraient régir une telle étude. Il s'agirait de comparer, comme dans l'expérience présentée ici, le potentiel acquisitionnel de différentes tâches qui se 
distingueraient selon qu'elles remplissent ou non - ou selon des degrés plus ou moins importants - les trois critères précédemment établis, à savoir :

- le degré de collaboration nécessaire à la résolution de la tâche ;

- la nécessité ou non de traduire sous forme linguistique l'information pertinente pour la résolution de la tâche (tâches à contenu iconographique versus tâches à contenu textuel) ;

- le degré de précision descriptive exigé.

La tâche, utilisée dans l'étude de Smith (2003), de remise en ordre des images (tâche "puzzle", que nous avons nous-mêmes utilisée lors d'une expérience préparatoire), nous semble, tout comme la tâche de comparaison entre les deux images, remplir ces conditions. L'expérience pourrait consister à la comparer à d'autres tâches qui ne remplissent pas ces conditions (ou qui les remplissent à un degré moindre), et par exemple à une tâche dont le principe est analogue mais qui ne remplit pas l'un des trois critères énoncés. On pourrait ainsi comparer une tâche de remise en ordre d'images avec une tâche de comparaison entre deux images, mais en modifiant légèrement le principe de résolution d'une des deux tâches ou son contenu (les images), de manière à amoindrir la précision descriptive, ou le degré de collaboration, requis pour sa résolution.

\section{Utilité et légitimité de l'approche quantitative}

En guise de conclusion, nous aimerions proposer quelques éléments de réflexion sur l'utilité et la légitimité qu'il y a à recourir à une approche quantitative/statistique et expérimentale pour étudier le phénomène d'acquisition de $\mathrm{L}^{24}$, en ouvrant le questionnement sur des considérations plus épistémologiques.

Pourquoi chercher à quantifier les effets et à évaluer la probabilité de les observer de nouveau dans des conditions analogues? Pourquoi ne pas se contenter d'une approche purement descriptive et qualitative? En bref, pourquoi recourir ici à la méthodologie expérimentale des sciences empiriques?

Pour des raisons de scientificité tout d'abord. Si l'on adopte une conception poppérienne de la science (réfutationnisme), les propositions et modèles de la RAL sur les mécanismes de l'acquisition et les situations facilitant celle-ci doivent être réfutables, pour pouvoir être considérés comme des propositions scientifiques, et ils doivent donc a minima être testables empiriquement. On peut bien sûr adopter une autre conception de la scientificité, le cas échéant moins contraignante, insistant par exemple plus sur la rigueur des méthodes, modèles et formalismes utilisés. Mais - et c'est le second point sur lequel nous tenons ici à insister -, cette rigueur ne suffit pas à assurer un caractère général aux propositions et modèles qui sont élaborés, c'est-à-dire qu'elle ne garantit pas le caractère reproductible des phénomènes que l'on prétend faire apparaitre. Or, si la RAL désire que ses observations et modèles puissent servir à l'établissement de méthodes ou plus généralement donner lieu à des recommandations pour la didactique des L2, par principe la reproductibilité des effets de facilitation de certains phénomènes et mécanismes qu'elle observe doit être assurée. Si les analyses des productions langagières que propose la RAL ne doivent pas se restreindre à des approches qualitatives et descriptives, mais doivent également être de nature quantitative, c'est que ce mode d'évaluation autorise l'utilisation d'outils statistiques permettant d'évaluer la généralité, et pour le moins la reproductibilité des phénomènes observés (voir J-P. Narcy-Combes, 2005: 100-103; Grosbois, 2007 ; Plonsky \& Gass, 2011). Dit autrement : les modèles descriptifs qu'élabore la 
RAL pour rendre compte des processus cognitifs individuels, mais également extra- et interindividuels (communication et normativité sociale), ont besoin, pour être également des modèles prédictifs, de s'établir sur des données empiriques ayant fait l'objet d'un traitement statistique préalable, destiné à éprouver leur reproductibilité. C'est à cette seule condition que les modèles peuvent prétendre à une forme de généralité, voire d'universalité.

Certes, on pourra rétorquer à cela que la didactique des L2, entendue comme démarche de théorisation ou de conceptualisation de l'activité pédagogique, dans sa finalité, son objet et ses méthodes, n'est pas assimilable à la RAL, qui s'intéresse pour sa part aux mécanismes - cognitifs ou sociocognitifs - qui sous-tendent l'acquisition d'une L2. Mais ceci n'exclut pas que la RAL puisse constituer une voie d'accès privilégiée à des pratiques pédagogiques optimisées. Klein (1989) l'explique très bien :

«L'acquisition d'une langue, maternelle ou seconde est un processus naturel. L'enseignement des langues est une tentative d'intervention dans ce processus naturel pour l'optimiser. Il est clair que cette intervention peut d'autant mieux réussir que nous en savons plus sur les lois qui régissent le processus sous-jacent. C'est pourquoi il est important d'étudier ces lois. C'est l'objet de la recherche sur l'acquisition des langues » (5).

Si la mise en place de dispositifs d'enseignement-apprentissage efficaces pour le développement des compétences de l'apprenant en L2 requiert une compréhension - fûtelle plus pratique ou opératoire que conceptuelle - des mécanismes cognitifs, et nous ajouterons sociocognitifs, qui président à ce développement, la RAL peut en effet être considérée comme un prérequis à une didactique des langues efficace. On peut sans doute concevoir une didactique des L2 qui œuvrerait de manière purement "pratique ", sans s'appuyer sur une compréhension conceptuelle des mécanismes cognitifs responsables de l'acquisition. Cependant, on peut également défendre l'idée que seule une connaissance approfondie des mécanismes de l'acquisition peut offrir à la didactique des L2 de réellement optimiser ses méthodes et ses dispositifs d'enseignement-apprentissage ${ }^{25}$.

Cette problématique rejoint directement la question, soulevée par différents travaux ${ }^{26}$, et qui est en filigrane derrière l'étude présentée dans cet article, de savoir si les tâches d'interaction peuvent être construites de manière à pouvoir prédéterminer l'usage du langage. Le phénomène de développement linguistique se caractérisant, comme tout phénomène humain, par une grande variabilité, une complexité qu'il est parfois difficile d'apprécier, et qu'il est encore plus difficile de conceptualiser de manière à en canaliser l'apparition et le déroulement, l'opportunité que nous fournissent les méthodes de la psychologie expérimentale de quantifier la reproductibilité des effets observés ne saurait être négligée.

BIBLIOGRAPHIE

Arditty, J. \& M-T. Vasseur (1999) Interaction et langue étrangère : présentation, in Arditty J. \& M.T. Vasseur (eds) Langages, 134, p. 3-19. 
Bange, P. (1990) A propos de la structure de l'interaction, in Conein, B., M. de Fornel, L. Quéré (éds) Les formes de la conversation, Réseaux, CNET, p. 51-68.

Behrent, S. (2007) La communication interalloglotte. Communiquer dans la langue cible commune, Paris : L'Harmattan, Coll. Espaces discursifs.

Benoît, W. (2004) L'approche par tâches dans l'apprentissage de l'anglais de spécialité : opérationnalisation contrôlée dans l'enseignement supérieur, Thèse de doctorat, Université de Nantes.

Braidi, S. (2002) Reexamining the role of recasts in NS/NNS interactions, Language Learning, 52, 1, p. 1-42.

Bruner, J. (1983) Le développement de l'enfant. Savoir faire, savoir dire. Paris : PUF.

Bygate, M., Skehan, P., Swain, M. (éds.) (2001). Researching pedagogic tasks: Second language learning, teaching and testing. Harlow : Longman.

Cosereanu, E. (2008). Interactions langagières : entre perception par les apprenants et analyse de corpus par les chercheurs, Les Cahiers de l'ACEDLE, 5, p. 129-150 (disponible sur : http:// acedle.org/IMG/pdf/Cosereanu_Cah5-1.pdf).

Cosereanu, E. (2009) Corrections entre pairs et interactions synchrones pour l'apprentissage du français langue étrangère, Les Cahiers de l'APLIUT, « La correction dans l'enseignement des langues de spécialité », 28, 3, p. 39-54.

Cosereanu, E. (2010a) Le rôle de la négociation et de la rétroaction corrective dans l'acquisition des langues secondes. La situation des tâches d'interaction en communication synchrone étudiée sur le cas du français langue étrangère, Thèse de doctorat, Université de Technologie de Compiègne, éd. ANRT, 2011, $880 \mathrm{p}$.

Cosereanu, E. (2010b) L'influence du contexte sur le potentiel acquisitionnel des tâches d'interaction : le cas du contexte sociolinguistique, in Foucher A.-L., M. Pothier, C. Rodrigues, V. Quanquin (dir). La tâche comme point focal de l'apprentissage. Actes du $2^{\text {ème }}$ colloque international Tidilem (Tice et Didactique des Langues Étrangères et Maternelles), 10-11 juin 2010, ClermontFerrand (disponible sur : http://edutice.archives-ouvertes.fr/docs/00/49/50/54/PDF/ Cosereanu.pdf).

Cosereanu, E. (2010c) Transferts interlinguistiques dans des tâches d'écart d'information chez des apprenants de FLE, Les Cahiers de l'ACEDLE, 7, 2, p. 5-36 (disponible sur : http://acedle.org/ spip.php?article3095).

De Pietro J.-F., Matthey M., Py B. (1989) Acquisition et contrat didactique : les séquences potentiellement acquisitionnelles de la conversation exolingue, in Weil D. \& H. Fugier (eds) Actes du 3 e colloque régional de linguistique, Université des sciences humaines et Université Louis Pasteur, Strasbourg, p. 99-124.

De Pietro, J-F. (1988) Vers une typologie des situations de contact linguistique, Langage et société, 43, p. 65-89.

Dewaele J. M. \& A. Furnham (2000) Personality and Speech Production: A Pilot Study of Second Language Learners, Personality and Individual differences, 28, p. 355-365.

Ellis, R. (1994) The study of Second Language Acquisition, Oxford: Oxford University Press.

Ellis, R. (2003) Task-Based Language Learning and Teaching, Oxford: Oxford University Press.

Gass S, Mackey A., L. Ross-Feldman (2005) Task-Based Interactions in Classroom and Laboratory Settings, Language Learning, 55, 4, p. 575-611.

Gass, S. (1997) Input, interaction, and the second language learner, Mahwah, NJ: Erlbaum. 
Grosbois, M. (2007) Didactique des langues et recherche expérimentale, Les Cahiers de l'Acedle, 4, p. 65-83 (disponible sur http://acedle.u-strasbg.fr/).

Hulstijn, J. (1989b) Implicit and incidental second language learning: experiments in the processing of natural and partly artificial input, in Dechert H. W. and M. Raupach (éds.) Interlingual processes, Tübingen: Gunter Narr. Verlag, p. 49-73.

Hulstijn,J. (1989a) A cognitive view of interlanguage variability, in Eisenstein M. R. (éd.) The Dynamic interlanguage: Empirical Studies in second language acquisition, New-York: Plenum, p. 17-31.

Klein, W. (1989) L'acquisition de langue étrangère, Armand Colin : Paris.

Lee, K.W. (2000) English Teachers' Barriers to the Use of Computer-assisted Language Learning, The Internet TESL Journal, 6, 12 (disponible sur http://iteslj.org/).

Long M.H. \& P. Robinson (1998) Focus on form: Theory, research, and practice, in Doughty C. \& J. Williams (éds.) Focus on form in classroom second language acquisition, New York: Cambridge University Press, p. 15-41.

Long, M.H. (1983) Linguistic and Conversational Adjustements to Non-native Speakers, Studies in Second language Learning, 5, p. 177-193.

Long, M.H. (1996). The role of the linguistic environment in second language acquisition, in Ritchie W.C. \& T.K. Bhatia (éds.) Handbook of second language acquisition, New York: Academic Press, p. 413-468.

Lyster, R. \& H. Mori (2006) Interactional feedback and instructional counterbalance, Studies in Second Language Acquisition (SSLA), 28, p. 269-300.

Narcy-Combes, J-P. (2005) Didactique des langues et TIC : vers une recherche-action responsable, Paris : Ophrys.

Narcy-Combes, M-F. (2010) Pour une méthodologie de description des profils d'apprenants : contexte et croisement des données, in Publication Plidam, Profils et parcours d'apprenant, compétence d'enseignant : quelles méthodologies? Éditions des Archives Contemporaines.

Pekarek, S. (2000) Approches interactionnistes de l'acquisition des langues étrangères : concepts, recherches, perspectives, Acquisition et interaction en langue étrangère (Aile), 12, p. 3-26.

Pekarek, S. (1999) Leçons de conversation. Dynamiques de l'interaction et acquisition de compétences discursives en classe de langue seconde, Fribourg : Éditions universitaires.

Pellettieri, J. (1999) Why talk? Investigating the role of task-based interaction through synchronous network-based communication among classroom learners of Spanish, thèse de doctorat, Université de Californie (Davis).

Pica, T. (1994) Research on negotiation: What does it reveal about second-language learning conditions, processes, and outcomes? Language Learning, 44, p. 403-527.

Plonsky, L. \& S. Gass (2011) Quantitative research Methods, Study Quality, and Outcomes: The Case of Interaction Research, Language Learning, 61, 2, p. 325-366.

Porquier R., \& B. Py (2004) Apprentissage d'une langue étrangère : Contextes et discours, Paris : Didier. Prabhu, N.S. (1987) Second Language Pedagogy, Oxford: Oxford University Press.

Py, B (1990) Les stratégies d'acquisition en situation d'interaction, in Gaonac'h D., D. Mac Nally, M.F. Ballaire (éds) Acquisition et utilisation d'une langue étrangère. L'approche cognitive. Le Français dans le Monde, Recherches et acquisition, Paris : Edicef, p. 81-88. 
Py, B., Matthey, M., De Pietro, J-F. (1988) Acquisition et contrat didactique : les séquences potentiellement acquisitionnelles dans la conversation exolingue, in Actes du $3^{\text {ème }}$ colloque Régional de Linguistique, Strasbourg, Université de Sciences Humaines et Université Louis Pasteur, Strasbourg, p. 99-119.

Schachter, J. (1984) A universal input condition, in Rutherford, W. (éd.), Universals and second language acquisition, Amsterdam: John Benjamins.

Schmidt, R. \& S. Frota (1986) Developing basic conversational ability in a second language: A case study of an adult learner of Portuguese, in Day R.R. (éd.) Talking to learn. Conversation in second language acquisition, Cambridge, MA: Newbury House Press, p. 237-326.

Schmidt, R. (1990) The role of consciousness in second language learning, Applied Linguistics, 11, p. 129-158.

Schmidt, R. (1995) Consciousness and foreign language learning: A tutorial on the role of attention and awareness in learning, in Schmidt R. (éd.) Attention and awareness in foreign language learning, Technical Report n 9, Honolulu, Hawaï: Université de Hawaï, p. 1-63.

Skehan, P. (1998) A Cognitive Approach to Language Learning, Oxford: Oxford University Press.

Smith, B. (2003) Computer-Mediated negotiated interaction: an expanded model, The Modern Language Journal, 87, p. 38-57.

Tatawy, M. E. (2002) Corrective Feedback in Second Language Acquisition, Working Papers in TESOL and Applied Linguistics, 2, 2, Teachers College, Columbia University. (disponible sur http:// journals.tc-library.org/index.php/tesol/issue/view/3)

Varonis, E.M. \& S. Gass (1985) Non-Native/Non native Conversations : A Model of Negotiation of Meaning, Applied Linguistics, 6, p. 71-90.

Véronique, D. (1992) Recherches sur l'acquisition des langues secondes : un état des lieux et quelques perspectives, Aile, 1, p. 5-37.

Véronique, D. (1997) La didactique des langues et des cultures face à l'apprenant de langues étrangères et à ses activités d'appropriation, in Galisson R. \& C. Puren (éds.) Études de linguistique appliquée, 105, p. 95-113.

Véronique, D. (2000) Recherches sur l'apprentissage des langues étrangères : friches et chantiers en didactique des langues étrangères, in Véronique, D. (éd.) Études de linguistique appliquée, 120, p. 405-417.

Véronique, D. (éd.) (2009) L'acquisition de la grammaire du français, langue étrangère, Paris, Didier. White, L. (1989) Universal Grammar and Second Language Acquisition, Amsterdam : John Benjamins. White, L. (1996) Universal Grammar and second language acquisition : Current trends and new directions, in Ritchie W.C. \& T.K. Bhatia (éds.) Handbook of second language acquisition, p. 85-120, New York : Academic Press.

Willis, J. (1996) A Framework for Task-Based learning, Harlow : Longman.

\section{NOTES}

1. Toutes les langues acquises ou en cours d'acquisition par l'apprenant, hormis la langue maternelle ou de première socialisation, sans ordre chronologique particulier (L2, L3,...Ln).

2. Voir Schmidt \& Frota (1986). 
3. Expression qui traduit les formules anglaises « positive evidence » et «negative evidence ». Il nous semble qu'on pourrait également traduire le terme anglais «evidence» par celui d'« échantillon », pour marquer l'idée que c'est bien un exemplaire de la norme acceptée en langue-cible qui est fourni au locuteur.

4. Voir par exemple Long (1996) ou Tatawy (2002). Plus largement, on peut se référer à Gass et al. (2005) qui expliquent que les trois caractères suivants ont été reconnus comme facilitant l'ALS dans la littérature de spécialité : 1. la négociation du sens 2. les épisodes relatifs au langage ( language related episodes) ; 3. la rétroaction corrective implicite (recast) (Gass et al., $2005: 585$ ).

5. Ou encore: « [Negociation of meaning is] the modification and restructuring of interaction that occurs when learners and their interlocutors anticipate, perceive, or experience difficulty in message comprehensibility » (Pica, 1994: 494, cité dans Braidi, 2002: 3).

6. «[...] negotiation for meaning, and especially negotiation work that triggers interactional adjustments by the native speaker or more competent interlocutor, facilitates acquisition because it connects input, internal learner capacities, particularly selective attention, and output in productive ways » (Long, 1996: 451-452).

7. Il s'agit de tâches d'interaction dont la résolution nécessite un transfert d'informations entre les locuteurs, ceux-ci disposant d'informations différentes au commencement de la tâche. Les informations de chacun doivent être mises en commun, comparées et confrontées pour que la tâche puisse être résolue. Il s'agira par exemple de tâches où les apprenants doivent trouver des différences entre des images dont chacun possède une version différente, ou de tâches de remise en ordre d'une histoire en images, dont chacun possède une partie. Pour plus de détails, voir Prabhu (1987), R. Ellis (2003), Cosereanu (2008, 2009, 2010a, 2010b, 2010c).

8. Cadre Européen Commun de Référence pour les langues, voir < http://www.coe.int/t/dg4/ linguistic/cadre_fr.asp>

9. Cosereanu (2008, 2009, 2010a, 2010b, 2010c).

10. Terme composé de clavier et bavardage, synonyme de chat.

11. Le document est disponible sur le site Internet du Conseil de l'Europe. http://www.coe.int/t/ dg4/linguistic/source/framework_FR.pdf

12. Voir Cosereanu (2008).

13. Pour plus de détails, voir Cosereanu (2010).

14. Également reprise par R. Ellis (2003).

15. Nous avons cherché ailleurs à évaluer l'impact d'autres variables sur les communications mises en place lors des tâches, en particulier : 1) la modalité communicationnelle utilisée ; 2) l'origine sociolinguistique des apprenants (leur langue maternelle ou de première socialisation) et les langues qu'ils maîtrisent déjà (les autres langues apprises que la L2 et la langue maternelle) ; 3) le contexte linguistique dans lequel a lieu l'apprentissage de la L2 (contexte homoglotte ou hétéroglotte). Voir nos autres travaux, cités plus haut, pour une présentation de ces résultats.

16. Voir section 2 .

17. Cet épisode de communication est également un exemple de contrat didactique, car l'apprenante B.P. endosse un rôle de tutrice en expliquant à son interlocuteur A.C. la règle grammaticale présidant à l'usage de l'adjectif.

18. Voir Cosereanu (2009) pour des exemples d'épisodes de communication où apparaît clairement cette démarche de tutorat.

19. Voir également l'étude de Varonis \& Gass (1985).

20. L'information est en effet déjà textuelle.

21. Cf. Smith (2003: 42).

22. Nous reprenons ici, en les synthétisant, des idées développées dans une étude antérieure (Cosereanu, 2008).

23. Cf. R. Ellis, $2003: 86$. 
24. On peut d'emblée mentionner ici l'étude menée par Plonsky \& Gass (2011), qui apporte des éléments essentiels sur cette question. Les auteurs ont cherché à évaluer si les études quantitatives réalisées depuis 30 ans dans le domaine de la RAL de facture interactionniste ont été menées en accord avec les normes de qualité méthodologique fixées dans les sciences sociales. Ils montrent que d'importantes améliorations méthodologiques ont été faites, et suggèrent l'existence d'une relation entre la qualité méthodologique des études et leurs résultats. 25. Pour une discussion de cette question, voir Véronique $(1997: 103)$ et Véronique $(2000: 406$ et suivantes).

26. Voir en particulier Willis (1996); Skehan (1998); Lee (2000); Bygate et al. (2001); R. Ellis (2003).

\section{RÉSUMÉS}

Nous présentons ici une étude expérimentale destinée à évaluer le potentiel acquisitionnel de différentes tâches d'interaction réalisées en binômes par des apprenants de français langue étrangère. Après avoir rappelé les principes du paradigme interactionniste, et sa conception du rôle de la correction et des tâches, nous décrivons le protocole de notre étude expérimentale, ainsi qu'une synthèse et une discussion de ses principaux résultats. Nous proposons enfin une discussion générale sur le rôle que l'expérimentation et les analyses quantitatives sont susceptibles de jouer dans la recherche en acquisition des langues et la didactique des L2. Nous défendons à ce titre l'idée que les analyses des productions langagières ne doivent pas se restreindre à des approches qualitatives et descriptives, mais qu'elles doivent également être de nature quantitative.

We present an experimental study designed to assess the potential for acquisition of different interaction tasks performed in pairs by learners of French as a foreign language. After recalling the principles of interactionist paradigm, and his conception of the role of the corrective feedback and the tasks, we present our experimental study protocol, and a summary and discussion of key results. We finally propose a general discussion of the role that experimentation and quantitative analysis can play in the research in second language acquisition. In this respect we defend the idea that analysis of language production should not be restricted to qualitative and descriptive analysis, but must also be quantitative.

\section{INDEX}

Keywords : research in second language acquisition, interaction tasks, negotiated interactions with potential for acquisition, experimental methodology, quantitative/qualitative analysis

Mots-clés : recherche en acquisition des langues secondes, tâches d'interaction, séquences potentiellement acquisitionnelles, méthodologie expérimentale, analyse quantitative/qualitative 


\section{AUTEUR}

\section{ELENA COSEREANU-DECLERCK}

DILTEC, EA 2288 (Université Sorbonne Nouvelle - Paris 3)

COSTECH, EA2223 (Université de Technologie de Compiègne)

elena.cosereanudeclerck@gmail.com 